\title{
Le peptide inhibiteur du diazepam
}

\section{Les nouvelles de ce numéro ont été préparées par : J. -C. Dreyfus} M.-H. Loucheux-Lefebvre

A. Kahn

D. Labie
1. Mocchetti I, Einstein R, Brosius J. Putative diazepam binding inhibitor peptide : cDNA clones from rat. Proc Natl Acad Sci USA 1986 ; 83 : 7221-5.

2. Gray PW, Glaister D, Seeburg PH, Guidotti A, Costa E. Cloning and expression of cDNA for human diazepam binding inhibitor. Proc Natl Acad Sci USA 1986 ; 83 : 7547-51. 3. Ferrero P, Santi MR, Conti-Tronconi B, Costa E, Guidotti A. Study of an octodecaneuropeptide derived from diazepam binding (DBI) : biological activity and presence in rat brain. Proc Natl Acad Sci USA $1986 ; 83$ : 827-31.

$\mathrm{m} / \mathrm{s} n^{\circ} 1$ ool. 3, janvier 87
L'acide gamma aminobutyrique (GABA) est le principal neurotransmetteur à action inhibitrice du système nerveux central des mammiferes. Son récepteur est complexe, il contient des sous-unités qui peuvent lier le GABA, mais aussi les benzodiazépines et les bêta carbolines. Les benzodiazépines accentuent l'action inhibitrice du GABA, les carbolines s'y opposent. La découverte de ligands endogènes pour les récepteurs des opiacés a conduit à postuler l'existence de phénomènes analogues dans le cas du récepteur du GABA. Un tel ligand a été identifié et appelé DBI (diazepam binding inhibitor). C'est une protéine d'environ 10000 Daltons, capable de déplacer in vitro benzodiazépines et $\beta$ carbolines liées à des membranes cérébrales. On a aussi observé leur présence dans nombre de régions du système nerveux central par des méthodes immunologiques.

Afin de mieux comprendre le mode de biosynthèse et le rôle du DBI, deux groupes américains en ont entrepris le clonage chez le rat [1] et chez l'homme [2], en partant dans les deux cas d'oligonucléotides de synthèse. La séquence complète de l'ADN complémentaire a été analysée, permettant de déduire celle des acides aminés. On remarque que le DBI du rat est nettement plus court ( 87 aa) que celui de l'homme (104 aa). Il ne possède pas de peptide signal, ce qui le différencie des neuropeptides classiques. Le nombre de bandes (au moins cinq) révélées par la méthode de Southern, avec plusieurs enzymes de restriction, permet de penser qu'il existe plusieurs gènes. Il est toutefois probable qu'un seul d'entre eux est fonctionnel, ou tout au moins fortement prédominant, car la méthode des Northern blots ne montre qu'une bande d'ARN messager, de 600 à 700 bases.

C'est le dosage de l'ARN messager par hybridation spécifique qui devait fournir les renseignements les plus intéressants. Comme prévu il est abondant dans le cerveau mais des études préliminaires par hybridation in situ montrent une répartition hétérogène avec des zones très enrichies. Cependant on en trouve aussi, à des concentrations élevées, dans des tissus non nerveux, surtout foie, rein, cœur et testicule, alors que le rôle éventuel du DBI dans ces organes n'est pas actuellement élucidé.

Une des hypothèses soulevées [2] est que le DBI, tel qu'il est synthétisé initialement, ne serait pas lui-même le facteur actif ; il serait secondairement scindé en produits de dégradation. Ferrero et al. [3] ont obtenu par hydrolyse trypsique du DBI humain un peptide de 18 acides aminés, plus actif pour déplacer la $\beta$ carboline que le DBI luimême. Il n'existe pas de preuve formelle que cette dégradation ait lieu in vivo. Si elle est confirmée, on peut émettre l'hypothèse qu'un peptide de 104 acides aminés, qui donne naissance à un peptide actif de taille très inférieure, pourrait également, par d'autres parties de sa molécule, être le précurseur d'autres peptides encore à découvrir, notamment dans les tissus qui ne font pas partie du système nerveux central. Malgré l'intérêt des résultats déjà acquis, le DBI et ses modalités d'action ont sans doute encore des secrets à révéler.

J.-C. D.

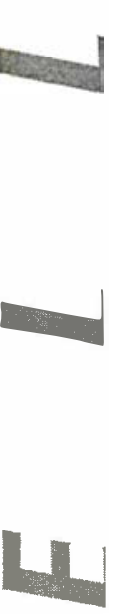

